Tropical Journal of Pharmaceutical Research April 2014; 13 (4): 511-518

ISSN: $1596-5996$ (print); 1596-9827 (electronic)

(C) Pharmacotherapy Group, Faculty of Pharmacy, University of Benin, Benin City, 300001 Nigeria.

All rights reserved.

Available online at http://www.tjpr.org

Original Research Article

http://dx.doi.org/10.4314/tjpr.v13i4.4

\title{
Molecular Dynamics and Bioactivity of a Novel Mutated Human Parathyroid Hormone
}

\author{
Yueshui Jiang ${ }^{1}$, Jiande $\mathrm{Gu}^{2}$, Jianyong Lei ${ }^{1}$, Yun Chen ${ }^{1}$ and Jian Jin ${ }^{1,3 *}$ \\ ${ }^{1}$ School of Pharmaceutical Sciences, Jiangnan University, 1800 Lihu Road, Wuxi, Jiangsu 214122, ${ }^{2}$ Drug Design \& Discovery \\ Center, Shanghai Institute of Materia Medica, Shanghai Institutes for Biological Sciences, Chinese Academy of Sciences, \\ Shanghai 201203, ${ }^{3}$ Institute of Health Sciences, Shanghai Institutes for Biological Sciences, Chinese Academy of Sciences \& \\ Shanghai Jiao-Tong University School of Medicine, 320 Yue-Yang Road, Shanghai 200031, PR China
}

*For correspondence: Email: jinjian31@126.com; Tel/Fax: +86 51085918219

Received: 3 May 2013

Revised accepted: 28 February 2014

\begin{abstract}
Purpose: To design and evaluate a novel human parathyroid hormone (hPTH) analog.

Methods: Mutation stability prediction was processed on hPTH, docked the mutant hPTH with its receptor, and then proceeded with molecular dynamics using Discovery Studio 3.5 software package for the complex. The bioactivity of the hPTH on the expression levels of the Rps27, RANKL and OPG genes were assessed in UAMS-32P cells.

Results: A three-site mutant, hPTH (R25Q:K26E:K27L), was obtained and MD trajectory analysis showed a decrease in the root mean square deviation by $51.95 \%$, in the radius of gyration by $3.57 \%$, and in the interaction energy by 10\%, compared with the wild-type hPTH. Bioactivity assessment demonstrated that this mutant stimulated the ratio of RANKL/OPG 30-fold higher than the wild-type.

Conclusion: We successfully designed a new hPTH mutant with a stable conformation and high bioactivity, and this may be useful for elucidating ligand-receptor recognition mechanism and discovering novel hPTH analogs.
\end{abstract}

Keywords: Parathyroid hormone, Mutation prediction, Molecular dynamics, RANKL/OPG, UAMS-32P cell

Tropical Journal of Pharmaceutical Research is indexed by Science Citation Index (SciSearch), Scopus, International Pharmaceutical Abstract, Chemical Abstracts, Embase, Index Copernicus, EBSCO, African Index Medicus, JournalSeek, Journal Citation Reports/Science Edition, Directory of Open Access Journals (DOAJ), African Journal Online, Bioline International, Open-J-Gate and Pharmacy Abstracts

\section{INTRODUCTION}

Parathyroid hormone (PTH) is an 84-amino-acid polypeptide that regulates extracellular calcium homeostasis and bone remodeling by direct action on the kidneys and bones [1]. PTH exerts its biological effects by binding to and activating the PTH receptor (PTH1R), which is highly expressed in PTH target tissues [2]. As a member of family B G protein-coupled receptor (GPCR), the PTH1R contains an $\mathrm{N}$-terminal extracellular domain (ECD) with three conserved disulfide bonds and a C-terminal domain with seven transmembrane helices [3]. The $\mathrm{N}$ - terminal 34-residue region of PTH (1-34) is capable of fully activating PTH1Rs to the same degree as PTH (1-84). Daily administration of either versions of PTH enhances bone formation, increases bone density, and decreases fracture risk in postmenopausal women $[4,5]$.

As a therapeutic peptide, human PTH (1-34) (hPTH) has natural limitations, such as low oral bioavailability, short half-life (only 5 min in serum) [6], high conformational flexibility (NMR studies revealed that hPTH (1-34) has a U-shaped tertiary structure [7], whereas its X-ray crystal structure is a single continuous helix [8]), and 
high synthesis and production costs (Forteo, a recombinant hPTH of Eli Lilly, costs US $\$ 16.70$ per day and US $\$ 6,100$ for a year of optimal therapy), which is a great therapeutic challenge to health-care systems [9]. Production of hPTH with a more stable structure and a higher PTH1R binding affinity is one way to overcome these shortcomings.

The hPTH (1-34) is defined as a 'two-domain model', in which the $\mathrm{N}$-terminal region (residues 1-14, hPTH (1-14)) recognizes and activates the PTH1R by attaching to the extracellular loops and the juxtamembrane regions of its transmembrane domain [10]. The C-terminal region (residues 15-34, hPTH (15-34)) comprises a high-affinity binding domain that binds to the PTH1R N-terminal ECD, resulting in high receptor affinity and specificity [11]. X-ray crystallography data indicate that hPTH (15-34) is a relatively independent and essential part of hPTH (1-34) [12]. Studies of deletions or mutations indicate that $\mathrm{hPTH}(15-34)$ is important for receptor binding, however, the adverse effects of substitutions in this region are not purely due to the disruption of tertiary interactions with hPTH (1-14) [13]. We therefore predicted a stable mutant of hPTH (15-34), verified its interaction with the PTH1R by molecular dynamics, synthesized mutated intact hPTH (1-34), and assessed its bioactivity.

\section{EXPERIMENTAL}

\section{Protein preparation and prediction of mutation stability}

We downloaded the pdb file of $\mathrm{PTH} 1 \mathrm{R} / \mathrm{hPTH}$ (15-34) (PDB: 3c4m) from the Protein Database Bank and corrected problems, such as nonstandard naming, structural disorders, missing side-chain or backbone atoms, protein residue connectivity, and bond orders, using the software of Discovery Studio (version 3.5, Accelrys Inc., San Diego, CA) clean protein tool. Chains A (PTH1R) and C (hPTH (15-34)) were preserved and the duplicated structure was removed as well as all hetatms that did not belong to the binding site. hPTH (15-34) molecule was then saved as a new file and applied CHARMm Polar $\mathrm{H}$ force field. Mutation stability was processed on single, double, and triple sites using Calculate Mutation Energy (Stability) protocol of Discovery Studio 3.5.

Calculate Mutation Energy (Stability) protocol evaluates the effect of single-point mutations on protein stability. It performs amino-acid scanning mutagenesis on a set of selected amino-acid residues by mutating each of them to one or more specified amino-acid types. The energy effect of each mutation on the protein stability (mutation energy, $\Delta \Delta \mathrm{Gmut}$ ) is calculated as the difference of the free energy of folding between the mutated structure and the wild type protein (Eq 1).

$\Delta \Delta \mathrm{Gmut}=\Delta \Delta \mathrm{G}$ fold $($ mutant $)-\Delta \Delta \mathrm{G}$ fold $($ wild type $) \ldots . .(1)$

where the folding free energy, $\Delta \Delta$ Gfold, is defined as free energy difference between the folded and unfolded states of the protein. The unfolded state is modeled as a relaxed peptide in extended conformation with the mutated residue in the center. This is used to account for the van der Waals and short-range electrostatic interactions between the mutated residue and the rest of the protein. In addition, a Gaussian random chain model is used to estimate the long range electrostatic interaction energy of the unfolded state. All energy terms are calculated by CHARMm and the electrostatic energy is calculated using a Generalized Born implicit solvent model.

\section{Molecular dynamics (MD) simulations}

Complex of the wild-type WT-hPTH (15-34) with PTH1R and the mutant MT-hPTH (15-34) with $\mathrm{PTH} 1 \mathrm{R}$ were used as MD simulation starting points. A full-atom system of PTH1R/WT-hPTH (15-34) in a solution box was constructed using the Discovery Studio 3.5 solvation protocol with default parameters. All ionizable residues were assigned to their respective charge status corresponding to $\mathrm{pH}$ 7.5. The complex was then solvated in a water box by the Explicit Periodic Boundary solvation model with a minimum distance of $7.0 \AA$ from the boundary. The cell shape was orthorhombic with $116.686 \times 88.7512 \times 65.3422 \quad \AA^{3}$ dimensions. Similarly, we also constructed an all-atom PTH1R/MT-hPTH (15-34) solution box system with $116.837 \times 88.7548 \times 65.5112 \quad \AA^{3}$ cell dimensions.

The system was subjected to the CHARMm force-field and relaxed by energy minimization (2000 steps of steepest descent and 2000 steps of conjugated gradient) with the harmonic restraint for the protein. The system was slowly driven from an initial temperature of $50 \mathrm{~K}$ to the target temperature of $300 \mathrm{~K}$ for $10 \mathrm{ps}$ and equilibration simulations were run for $10 \mathrm{ps}$. The MD simulations (production) were performed for 10 ns with the NPT system at a constant temperature of $300 \mathrm{~K}$ and the results were saved at a frequency of 1000 steps. All the other parameters were set as defaults. 


\section{MD trajectory analysis}

The MD trajectory was determined for structural properties, root mean-square deviation (RMSD), potential energy, and radius of gyration $(\mathrm{Rg})$ using the Discovery Studio 3.5 analyze trajectory protocol. The interaction energy between hPTH and the PTH1R was then calculated using the calculate interaction energy protocol. All parameters were set at defaults.

\section{Peptide synthesis}

The WT-hPTH (1-34) was obtained from Sigma Corp. (Santa Clara, CA). The MT-hPTH (1-34) was prepared by the solid-phase procedure and purified by gel filtration and sequential HPLC by GLS Biochem Co, Ltd. (Shanghai, China).

\section{Cell culture and bioactivity evaluation}

Confluent UAMS-32P cells stably expressing PTH1R were used to evaluate the bioactivity of WT-hPTH (1-34) and synthesized MT-hPTH (134). The effects of the hPTHs on the expression levels of the Rps27, RANKL and OPG genes were assessed using RT-PCR.

The UAMS-32P cell line was maintained in $\alpha$ minimal essential medium (Invitrogen, Carlsbad, CA) supplemented with $10 \%$ fetal bovine serum (FBS, HyClone, Logan, UT) and $1 \%$ each of penicillin, streptomycin, and glutamine. The vehicle was set as the negative control, along with WT-hPTH (1-34) as positive control, and the synthesized MT-hPTH (1-34) as the sample of interest. The UAMS-3P cells were treated with each agent at a concentration of $1 \times 10^{-7} \mathrm{~mol} / \mathrm{L}$ and total RNA was extracted from the cells after $24 \mathrm{~h}$.

\section{Statistical analysis}

The software suite of life science molecular design solutions Discovery Studio (version 3.5, Accelrys Inc., San Diego, CA) was used for protein design and molecular dynamics simulation. The analysis of the MD data was made using Origin Pro (version 9.0). Mean \pm standard error of mean (SEM) of the data were computed. The experimental data of bioactivity in Fig 3 were analyzed with GraphPad Prism (version 5.0). The level of significance was $p<$ 0.01 .

\section{RESULTS}

\section{hPTH mutation prediction}

We carried out full mutation scanning on Leu15 to Phe34 and obtained 380 single mutants, 1075 double mutants, and 637 triple mutants, then sorted them by their mutation energy. The two mutants with the lowest mutation energy of each type are listed in Table 1. Mutation energy prediction showed that the triple mutation had a much lower energy than the other two types. We then selected 5 mutants with the lowest mutation energy from each mutation type for further study (data not shown). In this study, the hPTH with the triple mutation of Arg25Gln, Lys26Glu, Lys27Leu was chosen for analysis.

\section{MD simulation trajectory}

The structural changes and the binding between $\mathrm{hPTH}$ and PTH1R were investigated by viewing the system potential energy, Rg, RMSD, and interaction energy (Fig 1). Statistical analysis was carried out for the last 5 ns of MD simulations to correlate the MD data with peptide characteristics (Table 2).

Values of RMSD, Rg, and potential energy evaluation demonstrated that the respective values became steady after $2 \mathrm{~ns}$ (Fig 1 ). The two complexes showed similar potential energy values, but PTH1R/MT-hPTH (15-34) reached equilibrium a little earlier than PTH1R/WT-hPTH (15-34) (Fig 1b), indicating that the mutated form quickly attained the stable state. The average RMSD of the mutant was $0.29 \mathrm{~nm}$, which dropped by $51.95 \%$ (Fig 1a). Rg decreased by $3.57 \%$, implying that the mutant was smaller and tighter than the wild-type (Fig 1c). Therefore, the

Table 1: Main characteristics of hPTH (15-34) mutation prediction

\begin{tabular}{|c|c|c|c|c|}
\hline Index & $\begin{array}{l}\text { Mutation } \\
\text { Type }\end{array}$ & Mutation Site & $\begin{array}{l}\text { Mutation Energy } \\
\text { (kcal/mol) }\end{array}$ & Effect \\
\hline 1 & Triple & C:ARG25.GLN,C:LYS26.GLU,C:LYS27.LEU & -10.3431 & Stabilizing \\
\hline 2 & Triple & C:SER17.ARG,C:GLU19.TYR,C:VAL21.ARG & -9.49557 & Stabilizing \\
\hline 3 & Double & C:GLU19.GLN,C:GLU22.CYS & -4.01122 & Stabilizing \\
\hline 4 & Double & C:ASP30.LEU,C:VAL21.ILE & -4.00888 & Stabilizing \\
\hline 5 & Single & C:ASP30.LEU & -2.26146 & Stabilizing \\
\hline 6 & Single & C:SER17.ARG & -2.10525 & Stabilizing \\
\hline
\end{tabular}



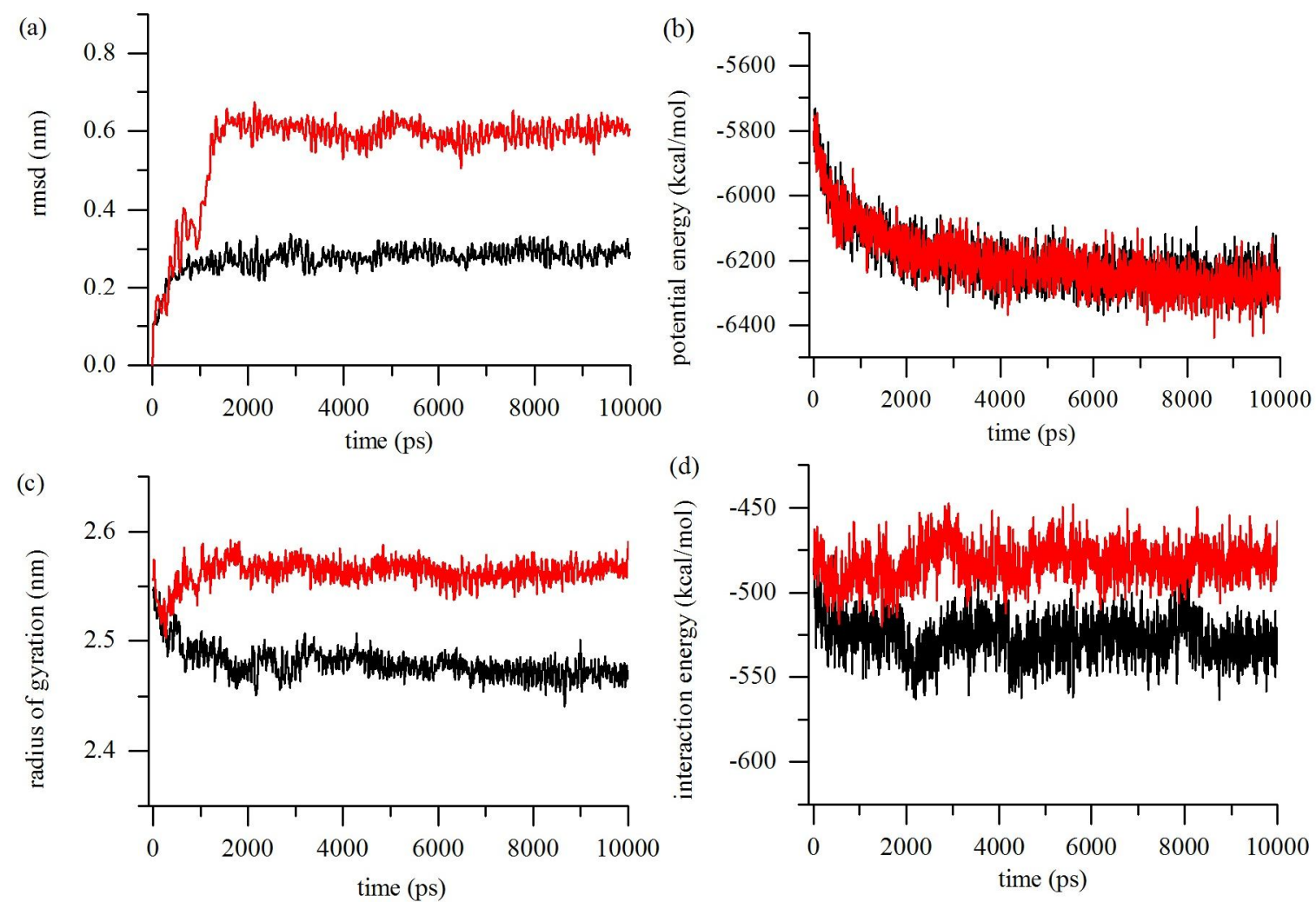

Fig 1: Molecular dynamics trajectory analysis of WT-hPTH (15-34) and MT-hPTH (15-34). Note: a = root meansquare deviation; $b=$ potential energy; $c$ = radius of gyration; $d=$ interaction energy. The trajectory was sampled every 1000 fs from the 10 ns of the MD, the WT-hPTH (15-34) was displayed as red and MT-hPTH (15-34) was black

Table 2: Comparison of statistics between WT-hPTH and MT-hPTH

\begin{tabular}{llll}
\hline Contribution & WT-hPTH & MT-hPTH & Comparison (\%) \\
\hline Potential energy (kcal/mol) & $-6262.21 \pm 1.68$ & $-6246.70 \pm 1.50$ & -0.25 \\
RMSD (nm) & $0.60 \pm 7.53 \mathrm{E}-04$ & $0.29 \pm 5.38 \mathrm{E}-04$ & -51.95 \\
Rg (nm) & $2.56 \pm 2.45 \mathrm{E}-04$ & $2.47 \pm 2.48 \mathrm{E}-04$ & -3.57 \\
Interaction energy $(\mathrm{kcal} / \mathrm{mol})$ & $-480.76 \pm 0.31$ & $-527.65 \pm 0.37$ & -9.75 \\
VDW $(\mathrm{kcal} / \mathrm{mol})$ & $-80.83 \pm 0.25$ & $-69.11 \pm 0.23$ & 14.50 \\
ELEC $(\mathrm{kcal} / \mathrm{mol})$ & $-399.92 \pm 0.39$ & $-458.54 \pm 0.44$ & -14.66 \\
H-bond (pairs) & $27.18 \pm 0.05$ & $30.22 \pm 0.07$ & -11.18 \\
\hline
\end{tabular}

conformation of PTH1R/MT-hPTH (15-34) was much more stable than that of PTH1R/WT-hPTH $(15-34)$.

The ligand-receptor interaction energy of WThPTH (15-34) and MT-hPTH (15-34) were shown in Fig 1d. The interaction energy of the PTH1R/WT-hPTH (15-34) was about $10 \%$ lower than that of PTH1R/MT-hPTH (15-34), demonstrating that the binding affinity between the mutant and the receptor was much higher than the wild type.

\section{MD simulation conformation}

In order to analyze the conformational changes between WT-hPTH (15-34) and MT-hPTH (1534), RMSF was calculated and the conformations with the lowest potential energy from the MD simulation trajectories were sampled.

The key residues of PTH1R involved in hPTH binding are shown in Fig 2. We found that the number of residues between MT-hPTH (15-34) and PTH1R-ECD was the same as that of WThPTH (15-34). There was a 3-residues difference: Thr33, Leu41, and Tyr136 in the wild- 
(a)

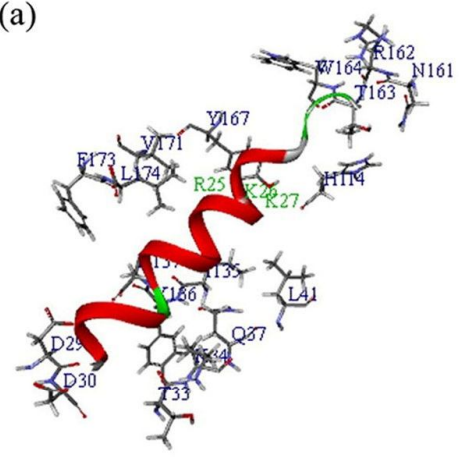

(c)

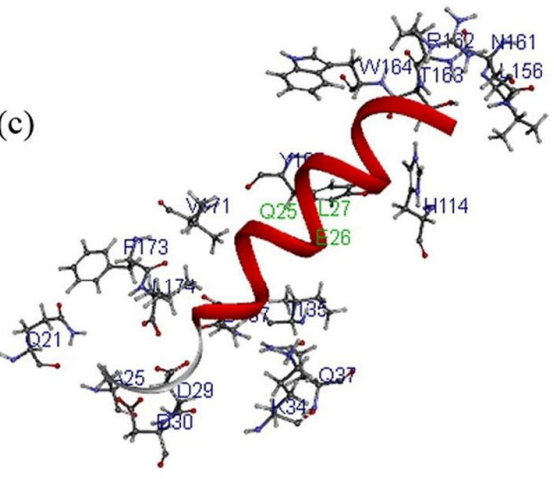

(e)

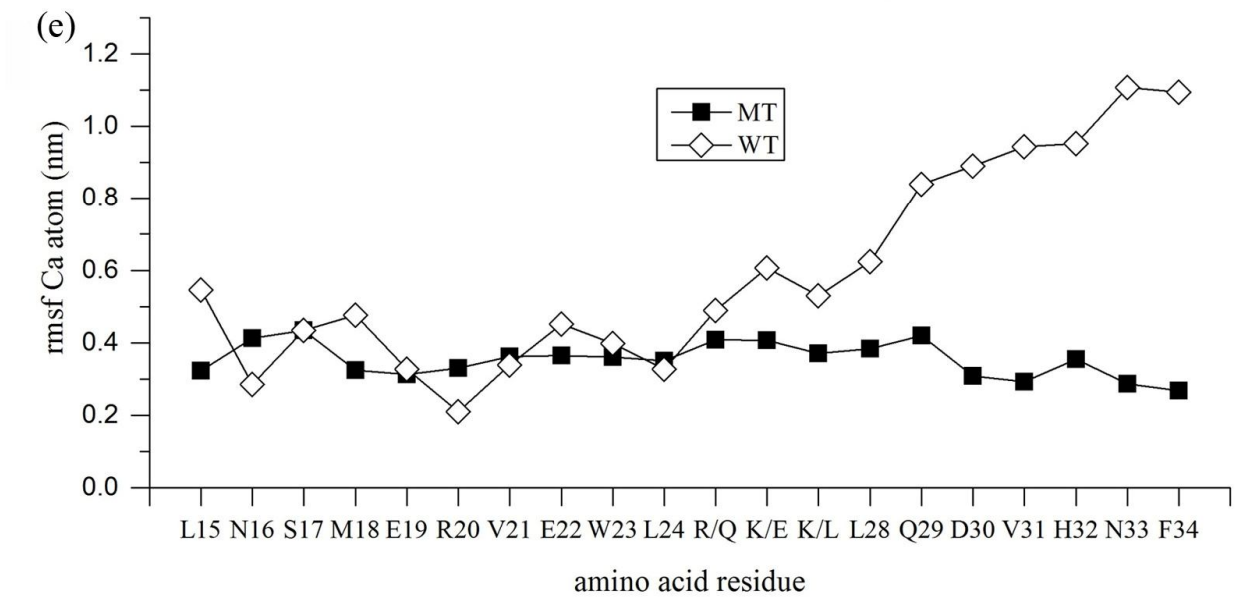

amino acid residue

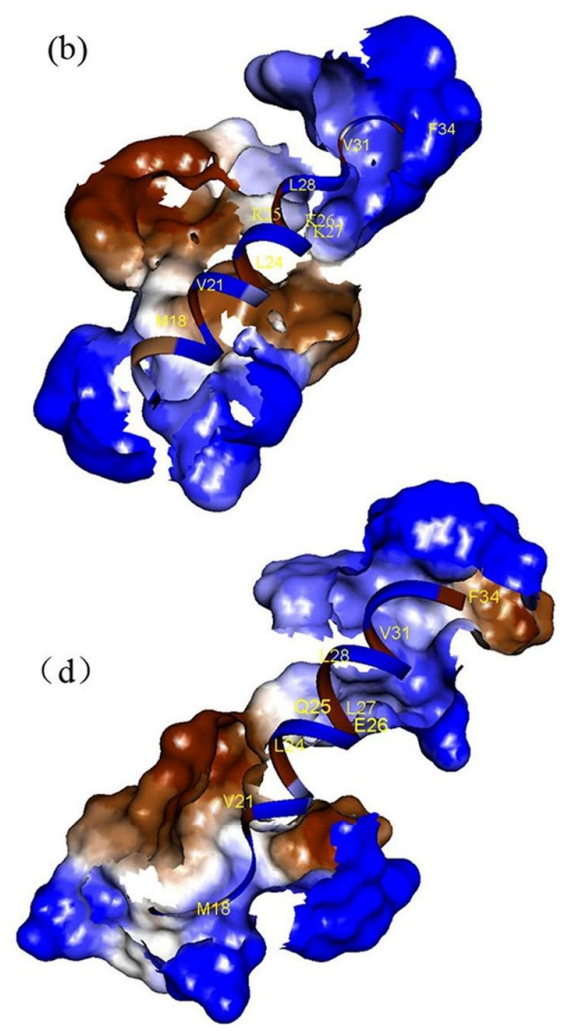

Fig 2: Residues on the interface of $\mathrm{PTH} 1 \mathrm{R} / \mathrm{hPTH}$ complex and their hydrophobicity. a and c showed the secondary structures of WT-hPTH (15-34) and MT-hPTH (15-34). Solid ribbon was applied for the display style and the colors were based on secondary structure: helices were red, beta sheets were cyan, turns were green, and coils were white. Residues on the PTH1R interface were shown as sticks and colored by element. $b$ and $d$. showed the hydrophobicity of WT-hPTH (15-34) and MT-hPTH (15-34). Residues on the PTH1R interface were also shown. Hydrophobic residues were brown, hydrophilic residues were blue, and neutral residues were white. e. rmsf of each residue of WT-hPTH (15-34) and MT-hPTH (15-34). The mutated sites were 25, 26 and 27, all amino acid residues were shown as one-letter code

type instead of GIn21, Ala25, and Leu156 in the mutant. These, along with the other 17 residues of the PTH1R (Asp29, Asp30, Lys34, GIn37, His114, Ile135, Asp137, Asn161, Arg162, Thr163, Trp164, Tyr167, Val171, Phe173, and Leu174) composed the interface of the two types of $\mathrm{hPTH}$ and acted as functional residues.
Hydrophobicity analysis showed that hydrophobic residues of MT-hPTH (15-34) lay along the amphipathic helix and faced a hydrophobic groove formed in the receptor interface. With the aid of hydrophobic force, Phe34 of the mutant was linked to Lys158. But Val31 and Phe34 of the wild-type did not establish contact with the receptor through 

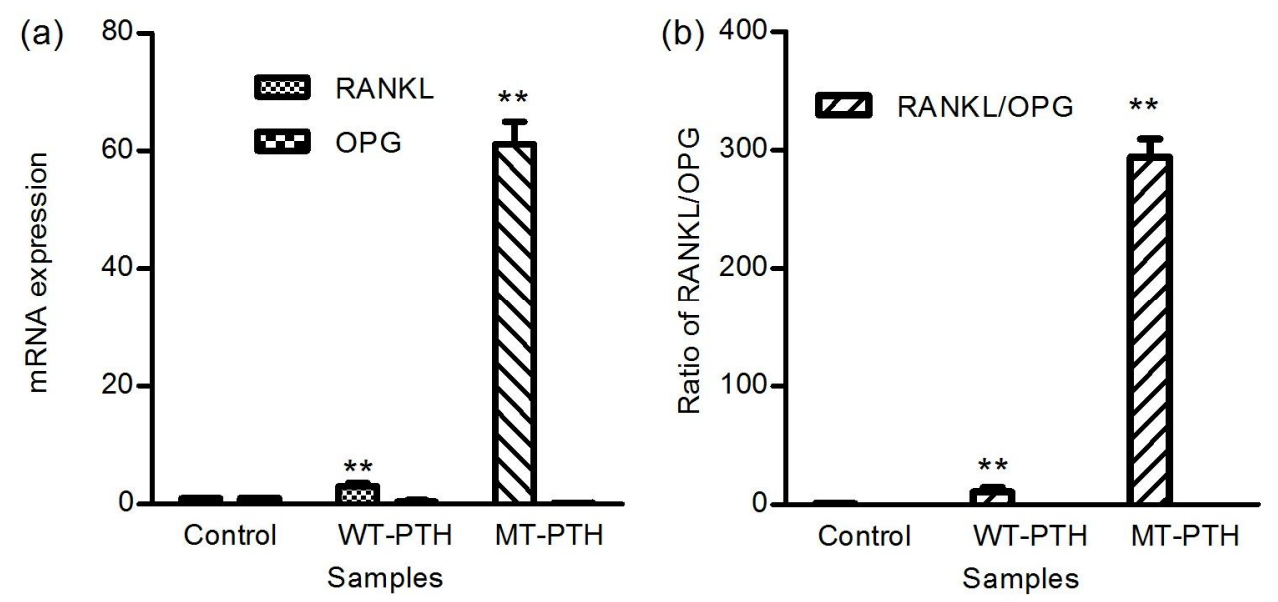

Fig 3: WT-hPTH (1-34), MT-hPTH (1-34) effects on mRNA levels of RANKL and OPG in UAMS-32P cells. The vehicle was set as the negative control, along with WT-hPTH (1-34) as positive control, and the synthesized MT$\mathrm{hPTH}(1-34)$ as the sample of interest. (a) the relatively mRNA expression level of RANKL and OPG vs Rps 27 gene; (b) the ratio of RANKL/OPG ( $\mathrm{n}=6,{ }^{*} p<0.05,{ }^{* *} p<0.01$, vs control)

hydrophobic interaction since they were in the turn or coil region of the peptide (Fig $2 b \& d$ ).

There was no change in the number of residues in the binding area, yet the MT-hPTH (15-34) interaction energy decreased by $\sim 10 \%$, while the ELEC decreased $14.66 \%$, and VDW increased by $14.50 \%$ compared with WT-hPTH (15-34). The average $\mathrm{H}$-bond formed between the mutant and PTH1R-ECD increased by $12 \%$ compared with the wild-type. The mutant had 3 more pairs of $\mathrm{H}$-bonds than the wild-type. This was likely to be an important reason for the higher binding affinity between the mutant and the receptor ECD and also gave an explanation of why they had a higher interaction energy.

To investigate the structural fluctuation, the RMSF of each residue of WT-hPTH (15-34) and MT-hPTH (15-34) was calculated (Fig 2e). The results showed that the RMSF values of the residues of the mutant were low and relatively constant, while those of the wild-type ranged from 0.2 to $1.1 \mathrm{~nm}$. The flexible residues in the 29-34 region were located at the C-terminal of the wild-type. We demonstrated that the Arg25GIn, Lys26Glu, and Lys27Leu mutations strengthened the stability of the $\mathrm{C}$-terminal region of hPTH.

\section{MT-hPTH (1-34) bioactivity}

PTH (1-34) stimulates the transcription of the RANKL gene and inhibits OPG gene transcription, increasing the RANKL/OPG ratio. The ratio of RANKL/OPG expression by osteoblasts is reported to be a key determinant of osteoclastogenic activity [14].

The vehicle (Control), WT-hPTH (1-34), and MThPTH (1-34) were subjected to RT-PCR assays; the RANKL and OPG gene expression in UAMS$32 \mathrm{P}$ cells was checked by comparison with Rps27 gene expression. The mutant of hPTH (R25Q:K26E:K27L) stimulated the expression of RANKL and decreased the expression of OPG to a high degree (Fig 3a). The RANKL/OPG ratio was $\sim 30$ times higher for hPTH (R25Q:K26E:K27L) than for WT-hPTH (Fig 3b).

\section{DISCUSSION}

Leu24 and Leu28 of hPTH (1-34) are located at the center of the hydrophobic interface [8]. Sitedirected mutagenesis studies on the C-terminal region of hPTH (1-34) have suggested that Leu24 and Leu28 are intolerant to mutation. When Leu24 and Leu28 are substituted by Glu, the receptor binding affinity decreases by $4000-$ and 1600 -fold, respectively [15]. In our mutation prediction, the mutation energy of Leu24 and Leu28 was high and the mutation effect was unstable (data not shown), which is largely in agreement with the previous study. Arg25, Lys26, and Lys27 located between the intolerant residues Leu24 and Leu28 are mutation-tolerant [16].

NMR studies confirmed that the 15-27 region of $\mathrm{hPTH}$ exists in a helical conformation and the 2834 region has a highly flexible structure [7]. Our RMSF analysis revealed that the 15-28 region had relatively constant fluctuate values, and the 
RMSF of the 28-34 region ranged from 0.2 to 1.1 $\mathrm{nm}$ (Fig 2e), which supported the NMR results, and gave a possible explanation of why the 2834 region did not inhibit radio-ligand binding at any concentration. When Arg25, Lys26, and Lys27 were substituted with GIn25, Glu26 and Leu27, the conformation of the 28-34 region changed from a random coil into a helix (Fig 2e). It appeared that mutation of these three residues affected the conformation of the 28-34 region. Meanwhile, the position of MT-hPTH (15-34) within the complex moved downwards with 3 different residues among the PTH1R/hPTH interface residues (Fig 2a \& b).

In addition, we found that the Lys26-Glu mutation led to the formation of two new $\mathrm{H}$-bond pairs. Lys27 is hydrophilic polar amino-acid, whereas its mutant residue Leu is hydrophobic and nonpolar, so this mutation increased the hydrophobic area between hPTH and its receptor (Fig 2b \& d). It is likely that the increase of $\mathrm{H}$-bond and hydrophobic force resulted in the enhancement of ligand-receptor interaction energy.

So far, mutagenesis studies of PTH have been carried out in PTH-specific binding sites and their interaction with its receptor $[11,17]$; however there are no reports on the prediction of $\mathrm{hPTH}$ multi-site mutations. We performed mutation prediction and obtained a triple hPTH mutant, hPTH (R25Q:K26E:K27L), which evidently increased the RANKL/OPG ratio by 30 times, but its mechanism of action remains unclear. These findings therefore present a new conceptual starting-point for unraveling the ligand-receptor recognition mechanism and consequently provide a guide to designing novel hPTH analogs.

\section{CONCLUSION}

PTH is a key regulator of calcium homeostasis and a major mediator of bone remodeling. Due to its natural limitations, we carried out mutation prediction and bioactivity evaluation to get a mutant with higher bioactivity and more structural stability. The ligand-receptor recognition mechanism was also investigated. As a result, we successfully designed a new hPTH mutant with a stable conformation and high bioactivity, which may be useful for elucidating the ligandreceptor recognition mechanism and discovering novel hPTH analogs.

\section{ACKNOWLEDGEMENT}

This work was supported by the National Natural Science Foundation of China (no. 81273437) and the Fundamental Research Funds for the Central Universities in China (JUDCF10065). The authors thank Dr. Iain C Bruce for thorough revision of the manuscript.

\section{REFERENCES}

1. Martin TJ, Allan EH, Caple IW, Care AD, Danks JA, Diefenbach-Jagger $H$, Ebeling PR, Gillespie $M T$, Hammonds G, Heath JA, et al. Parathyroid hormonerelated protein: isolation, molecular cloning, and mechanism of action. Recent Prog Horm Res 1989; 45: 467-506.

2. Juppner $H$, Abou-Samra AB, Freeman $M$, Kong $X F$, Schipani E, Richards J, Kolakowski LF, Jr., Hock J, Potts JT, Jr., Kronenberg HM, et al. A G proteinlinked receptor for parathyroid hormone and parathyroid hormone-related peptide. Science 1991; 254(5034): 1024-1026.

3. Gardella TJ, Juppner $H$. Molecular properties of the PTH/PTHrP receptor. Trends Endocrinol Metab 2001; 12(5): 210-217.

4. Neer RM, Arnaud CD, Zanchetta JR, Prince R, Gaich GA, Reginster JY, Hodsman AB, Eriksen EF, IshShalom S, Genant HK, et al. Effect of parathyroid hormone (1-34) on fractures and bone mineral density in postmenopausal women with osteoporosis. N Engl J Med 2001; 344(19): 1434-1441.

5. Moen MD, Scott LJ. Recombinant full-length parathyroid hormone (1-84). Drugs 2006; 66(18): 2371-2381.

6. Satterwhite J, Heathman M, Miller PD, Marin F, Glass EV, Dobnig $H$. Pharmacokinetics of teriparatide (rhPTH[1-34]) and calcium pharmacodynamics in postmenopausal women with osteoporosis. Calcif Tissue Int 2010; 87(6): 485-492.

7. Barden JA, Kemp BE. NMR solution structure of human parathyroid hormone (1-34). Biochemistry 1993; 32(28): 7126-7132.

8. Jin L, Briggs SL, Chandrasekhar S, Chirgadze NY, Clawson DK, Schevitz RW, Smiley DL, Tashiian $A H$, Zhang F. Crystal structure of human parathyroid hormone 1-34 at 0.9-A resolution. J Biol Chem 2000; 275(35): 27238-27244.

9. Berg C, Neumeyer K, Kirkpatrick P. Teriparatide. Nat Rev Drug Discov 2003; 2(4): 257-258.

10. Luck MD, Carter PH, Gardella TJ. The (1-14) fragment of parathyroid hormone (PTH) activates intact and amino-terminally truncated $\mathrm{PTH}-1$ receptors. $\mathrm{Mol}$ Endocrinol 1999; 13(5): 670-680.

11. Potetinova Z, Barbier JR, Suen T, Dean T, Gardella TJ, Willick GE. C-terminal analogues of parathyroid hormone: effect of C-terminus function on helical 
structure, stability, and bioactivity. Biochemistry 2006; 45(37): 11113-11121.

12. Pioszak AA, Xu HE. Molecular recognition of parathyroid hormone by its $G$ protein-coupled receptor. Proc Natl Acad Sci USA 2008; 105(13): 5034-5039.

13. Chen BY, Qu P, Tie R, Zhu MZ, Zhu XX, Yu J. Protecting effects of vasonatrin peptide against carbon tetrachloride-induced liver fibrosis. Regul Pept 2010; 164(2): 139-143.

14. Huang JC, Sakata T, Pfleger LL, Bencsik M, Halloran BP, Bikle DD, Nissenson RA. PTH differentially regulates expression of RANKL and OPG. J Bone Miner Res 2004; 19(2): 235-244.
15. Yu J, Zhu M, Fu Z, Zhu X, Zhao Y, Chen B. Vasorelaxing action of vasonatrin peptide is associated with activation of large-conductance $\mathrm{Ca}(2+)$-activated potassium channels in vascular smooth muscle cells. Physiol Res 2010; 59(2): 187-194.

16. Zhao HK, Chen BY, Chang R, Wang JB, Ni F, Yang L, Dong XC, Sun SH, Zhao G, Fang $W$, et al. Vasonatrin peptide, a novel protector of dopaminergic neurons against the injuries induced by n-methyl-4phenylpyridiniums. Peptides 2013).

17. Chen BY, Tie R, Qu P, Zhu MZ, Zhu XX, Jin J, Yu J. Vasonatrin peptide, a new regulator of adiponectin and interleukin-6 production in adipocytes. J Endocrinol Invest 2011; 34(10): 742-746. 